to the overlapping of non-communicable diseases (NCDs) and transmitted diseases (TDs) as the main causes of morbidity and mortality. This study describes the distribution of avoidable mortality from the Brazilian List of Preventability (LBE) among the elderly (60-75 years) in the cities of Belo Horizonte, Porto Alegre and Recife in the years 2003-2007. Data on elderly mortality were taken from the Mortality Information System (SIM). The profile of causes of death in the three cities showed no significant differences. In all of them, LBE preventable causes of deaths were more frequent-average of 2907 deaths (60\%). The NCDs accounted for 1248 deaths (83.2\%). Ischaemic heart diseases was the largest cause of NCDs. Among the TDs ( $\mathrm{n}=163$ deaths), respiratory infections were the main cause of death. Among causes avoidable by immunisation, TB was the most prevalent. Accidents and violence were 2.2 times more frequent among men. In the three cities, traffic accidents were also prominent. Preventable deaths predominated, especially NCDs, despite there also being a significant number of deaths from TDs. The use of the LBE showed the process of epidemiological transition in the three cities. I order to reduce premature mortality among the elderly, it is essential to invest in programs and actions promoting health and prevention of both NCDs and TDs.

\section{P2-349 USE OF INSECTICIDE TREATED NETS AMONG PREGNANT WOMEN ATTENDING ANTENATAL CARE AT A PRIMARY HEALTHCARE FACILITY KADUNA STATE, NIGERIA}

doi:10.1136/jech.2011.142976k.81

\begin{abstract}
${ }^{1}$ A A Abubakar, ${ }^{1}$ M N Sambo, ${ }^{1}$ S H Idris, ${ }^{1}$ K Sabitu, ${ }^{2} \mathrm{P}$ Nguku, ${ }^{1}$ S Seidu, ${ }^{1}$ A A Aliyu, ${ }^{1}$ A U Shehu. ${ }^{1}$ Ahmadu Bello University, Zaria, Kaduna State, Nigeria; ${ }^{2}$ Centers for Disease Control \& Prevention, Abuja, Nigeria
\end{abstract}

Introduction Malaria is an important public health problem in Nigeria. Pregnant women are among the high risk groups for developing malaria. Insecticide Treated Nets (ITNs) have been shown to be an effective means of preventing malaria. A study was undertaken to assess use of ITNs among pregnant women attending Antenatal care.

Methods A descriptive study was conducted. A sample of 330 pregnant women attending Antenatal clinic at a Primary Healthcare center were interviewed on history of fever, use of ITNs and use of other interventions to repel or kill mosquitoes.

Results The mean age of respondents was 25.25 years $( \pm 5.48)$. More than a third 127 (39\%) were in their third trimester of pregnancy. Most 273 (83\%) reported having had a fever during the index pregnancy. Only $43(13 \%)$ had any type of mosquito net. Overall 25 $(7.6 \%)$ had ITNs. Pregnant women with higher levels of education were more likely to have mosquito nets than those with lower levels of education (Secondary education or higher $72 \%$ vs Primary education 14\%). Most 324 (98\%) of the ANC attendees used other interventions to repel or kill mosquitoes, 181 (56\%) mainly used insecticide sprays.

Conclusion There is low utilisation of Insecticide Treated Nets by pregnant women attending Antenatal Care. Use of other interventions to kill or repel mosquitoes was higher. Antenatal Care provides an opportunity to create awareness and distribute Insecticide Treated Nets to pregnant women. Indoor Residual Spraying is likely to be acceptable as a means of vector control.

\section{P2-350 WILLINGNESS AND SELF-RATED COMPETENCE TO PROVIDE SMOKING CESSATION SERVICES BY FAMILY AND COMMUNITY MEDICINE DOCTORS IN A TERTIARY HOSPITAL IN NIGERIA}

doi:10.1136/jech.2011.142976k.82

A 0 L Adebiyi, E 0 Cadmus, 0 K Ige, E A Bamgboye. Nigerian Tobacco Control Research Group, College of Medicine, University of Ibadan, Ibadan, Oyo State, Nigeria

Background Patients advised to quit smoking by their physicians are more likely to quit than patients not receiving physician advice. However, most smokers do not receive this advice when visiting their physicians. This scoping study was to assess the willingness and self-rated competence of doctors to provide tobacco cessation services as a prelude to the establishment of a hospital based cessation service.

Methodology Self-administered semi-structured questionnaire was used to collect information from doctors working in the family medicine and community medicine departments of a teaching hospital.

Result Forty-one doctors participated in the study. Mean age of participants was $35.5 \pm 7.5$ years. Majority $(61.0 \%)$ were males and most $(78.1 \%)$ had worked $\leq 5$ years in the institution. About half $(51.2 \%)$ were aware of Nigeria tobacco decree, $14.5 \%$ were aware of WHO-FCTC and the 5As of smoking cessation. Only $4.9 \%$ had ever used the change model. Barriers to tobacco cessation activities in everyday practice included having no materials to hand out $(41.5 \%)$, lack of time $(29.3 \%)$, not knowing what to say or do $(14.6 \%)$; and where to send patients for counselling (31.7\%). Smoking cessation services were offered routinely in the clinics of $17.1 \%$ of respondents. Less than $10 \%$ believed they had excellent knowledge and skills in pharmacology of nicotine, motivating patients to quit and behavioural smoking cessation techniques. Seventy percent expressed willingness to learn more about these topics with majority $(53.7 \%)$ preferring the on-site continuing medical education mode.

Conclusion Training and creation of an enabling environment is needed to encourage doctors to practice tobacco cessation in their facilities.

\section{P2-351 IRON STATUS AND CARDIOVASCULAR DISEASE RISK IN BLACK SOUTH AFRICAN WOMEN: THE PURE STUDY}

doi:10.1136/jech.2011.142976k.83

1,20 Aderibigbe, ${ }^{1} \mathrm{P}$ Pisa, ${ }^{1} \mathrm{R}$ Mamabolo, ${ }^{1} \mathrm{~S}$ Kruger, ${ }^{1} \mathrm{H}$ Vorster, ${ }^{1} \mathrm{~A}$ Kruger. ${ }^{1}$ NorthWest University, Centre of Excellence for Nutrition, Potchefstroom, South Africa; ${ }^{2}$ National horticultural Research Institute, Ibadan, Nigeria

Objective To examine the associations between measures of iron status and cardiovascular disease (CVD) risk factors in South African women.

Design The study was cross-sectional in design. Demographic information and health history were obtained during individual interviews using validated questionnaires. Anthropometric indices, iron indices, blood pressure, blood glucose and lipid profiles were measured using standard procedures. Iron status was assessed using serum concentrations of ferritin, transferrin receptor and transferrin receptor to ferritin ratio.

Setting North West Province of South Africa.

Subjects 1262 apparently healthy black South African women (>35 years).

Results Associations between iron status parameters and CVD risk factors were generally weak $(r<0.3, p<0.01)$ and were not retained when age, BMI, smoking, alcohol consumption and $\mathrm{C}$ 